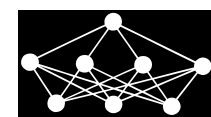

\title{
QUANTUM MULTIDIMENSIONAL MODELS OF COMPLEX SYSTEMS
}

\author{
M. Svítek*
}

\begin{abstract}
The paper presents a new methodology how to extend the well-known quantum model [2] with $(2 N-1)$ free parameters (moduli and phases) of wave probabilistic functions $\psi\left(A_{i}\right)$ assigned into events $A_{i}, i \in\{1,2, \ldots, N\}$ to $\frac{N \cdot(N+1)}{2}$ free parameters necessary for full $N$-dimensional representation of complex system. Our approach generally enables to include additional functions applied on events $A_{i}, i \in\{1,2, \ldots, N\}$. In the paper, we will demonstrate this mathematical instrument on additional wave probabilistic functions $\psi\left(A_{k} \cap A_{m} \cap \cdots \cap A_{n}\right)$ connected with macroscopic events' intersections $A_{k} \cap A_{m} \cap \cdots \cap A_{n}$ where $k, m, \ldots, n \in$ $\{1,2, \ldots, N\}$.
\end{abstract}

Key words: quantum system theory, quantum informatics, complex systems, information physics, extended quantum models, wave probabilistic functions, smart systems

Received: March 19, 2019

DOI: $10.14311 /$ NNW.2019.29.022

Revised and accepted: October 27, 2019

\section{Introduction}

A probability space consists of a sample space $S$ and a probability function $P(\cdot)$, mapping the events of $S$ to real numbers in $[0,1]$, such that $P(S)=1$, and if $A_{1}, A_{2}, \ldots, A_{N}$ is a sequence of disjoint events, then the sum rule is fulfilled:

$$
P\left(\bigcup_{i \in N} A_{i}\right)=\sum_{i \in N} P\left(A_{i}\right) .
$$

If the events $A_{1}, A_{2}, \ldots, A_{N}$ are not disjoint, the following (intersection and union) rules can be used:

$$
\begin{gathered}
P\left(A_{1} \cap A_{2} \cap \cdots \cap A_{n}\right)=P\left(A_{1}\right) \cdot P\left(A_{2} \mid A_{1}\right) . \\
\cdot P\left(A_{3} \mid A_{1} \cap A_{2}\right) \ldots P\left(A_{N} \mid A_{1} \cap \cdots \cap A_{N-1}\right), \\
P\left(A_{1} \cup A_{2} \cup \cdots \cup A_{N}\right)= \\
=\sum_{i-1}^{N} P\left(A_{i}\right)-\sum_{i<j}^{N} P\left(A_{i} \cap A_{j}\right)+\sum_{i<j<k}^{N} P\left(A_{i} \cap A_{j} \cap A_{k}\right)+\ldots \\
+\quad(-1)^{N-1} \cdot P\left(A \cap A \cap \cdots \cap A_{N}\right) .
\end{gathered}
$$

*Miroslav Svítek; Faculty of Transportation Sciences, CTU Prague, E-mail: svitek@fd.cvut. $\mathrm{Cz}$ 
Considering the basic laws of probability Eq. (3), we need generally $\frac{N \cdot(N+1)}{2}$ free parameters.

The main goal of the paper is to extend a quantum model [2] with $(2 N-1)$ free parameters in such a way that it could describe the system with more parameters. In this way, the curse of dimensionality can be overcome, at least in part, with the increasing dimensionality of the system, the number of required parameters for its full description rapidly increases. The Section 2 summarizes the basic definition and some features of quantum models. Section 3 shows the applicability limits of current quantum models. In Section 4, the new approach to quantum extended models is described together with illustrative examples presented in Section 5. Section 6 concludes the paper.

\section{Quantum models}

Let us suppose $N$ events $A_{i}, i \in\{1,2, \ldots, N\}$ with defined probabilities $P\left(A_{i}\right)$, $i \in\{1,2, \ldots, N\}$, and $N$ wave probabilistic functions:

$$
\psi\left(A_{i}\right)=\alpha_{i} \cdot e^{j \cdot v_{i}}=\sqrt{P(A)_{i}} \cdot e^{j \cdot v i}, i \in\{1,2, \ldots, N\},
$$

together with their superposition state $|\psi\rangle$ as a quantum object [3]:

$$
|\psi\rangle=\psi\left(A_{1}\right) \cdot\left|A_{1}\right\rangle+\psi\left(A_{2}\right) \cdot\left|A_{2}\right\rangle+\cdots+\psi\left(A_{N}\right) \cdot\left|A_{N}\right\rangle
$$

with moduli $\sqrt{P\left(A_{i}\right)}$ and phases $v_{i}$, where the reference phase assigned to event $A_{1}$ is chosen as $v_{1}=0$. The intersection and union rules for quantum models were defined in [4]:

$$
\begin{gathered}
P\left(\left|A_{1}\right\rangle \cup\left|A_{2}\right\rangle \cup \cdots \cup\left|A_{N}\right\rangle\right)=\left|\sum_{i=1}^{N} \psi\left(A_{i}\right)\right|^{2}, \\
P\left(\left|A_{r}\right\rangle \cap\left|A_{s}\right\rangle\right)=\lim _{\substack{P\left(A_{k}\right) \\
k \neq r, s}}\left[\psi^{*}\left(A_{r}\right) \cdot \psi\left(A_{s}\right)+\psi\left(A_{r}\right) \cdot \psi^{*}\left(A_{s}\right)\right],
\end{gathered}
$$

where symbol $\psi^{*}$ expresses a complex conjugate of $\psi$.

Quantum model Eq. (4) and (5) provides only $(2 N-1)$ parameters - moduli $\left|\psi\left(A_{i}\right)\right|$ and phases $\nu_{i}$ of wave probabilistic functions $\psi\left(A_{i}\right)=\left|\psi\left(A_{i}\right)\right| \cdot e^{j \cdot \nu i}$. Let us show the dimension limit of quantum model on following illustrative example.

Example 1 - N-dimensional distribution and its approximation by quantum model We will assume $N$-dimensional distribution for which we need to specify all the values of the $N \times N$ covariance matrix $\sigma_{i, j}$. Due to symmetry, we need only $\frac{N \cdot(N+1)}{2}$ parameters.

Since we cannot determine the covariance matrix $\sigma_{i, j}$ exactly, we therefore need to come up with an approximate description, a description that would require fewer parameters.

Instead of representing each quantity $\delta_{i}$ as an $N$-dimensional vector $a_{i}=$ $\left(a_{i, 1}, \ldots, a_{i, N}\right)$ corresponding to $\delta_{i}=\sum_{j=1}^{N} a_{i j} \cdot X_{j}$ where $\left\{X_{1}, \ldots, X_{N}\right\}$ are independent standard random variables, we select some value $k<<N$ and represent each quantity $\delta_{i}$ as a $k$-dimensional vector corresponding to $\delta_{i}=\sum_{j=1}^{k} a_{i j} \cdot X_{j}$. For $k=2$, the approximation leads to a Quantum model [1]. 


\section{Practical applications of quantum models}

In quantum (wave) approximation, only $(N-1)$ phase parameters among $N$ dimensional events $A_{i}, i \in\{1,2, \ldots, N\}$ are available. For simplicity, we will not be concerned by moduli $\left|\psi\left(A_{i}\right)\right|$ because they have no impact on phase parameters. Look at practical examples of quantum models' applicability.

\subsection{Ordering models}

Suppose the unique ordering of the events $\left\{A_{1}, A_{2}, \ldots, A_{N}\right\}$ where each index represents the event's order in sequence and the distance between two events $l, m$ is defined as $d_{l, m}=|l-m|$. In quantum notation, the phase difference $\nu_{l, m}=\nu_{l}-\nu_{m}$ represents correlation (link) between two events. The quantum (wave) model is fully applicable in case the correlation between events $l, m$ is dependent only on the distance between them $d_{l, m}=|l-m|$ and not on their position. For this example, the new phase parameters $\tilde{\nu}_{i}$ could be introduced:

$$
\begin{aligned}
\tilde{\nu}_{1} & =\nu_{2}-\nu_{1}=\nu_{3}-\nu_{2}=\ldots \nu_{N-1}-\nu_{N-2}=\nu_{N}-\nu_{N-1} \\
\tilde{\nu}_{2} & =\nu_{3}-\nu_{1}=\nu_{4}-\nu_{2}=\ldots=\nu_{N}-\nu_{N-2} \\
& \vdots \\
\tilde{\nu}_{N-1} & =\nu_{n}-\nu_{1} .
\end{aligned}
$$

From (8) it is clear that we can describe whole system by $(N-1)$ phase parameters $\left(\tilde{\nu}_{1}, \tilde{\nu}_{2}, \ldots, \tilde{\nu}_{N-1}\right)$. This model is typical for time-invariant subsystems [5] where the correlation is dependent only on the time differences between two realizations.

\subsection{Incremental models}

Let us suppose the existence of a reference event (a phase of event $A_{1}$ ) typically equal to $\nu_{1}=0$, from which we measure the correlations (links) to other events. In incremental model, due to additivity all other correlations could be computed from phases. For example, a phase difference between events $k$ and $(k+d)$ has to be equal to $\nu_{d}=\nu_{k+d}-\nu_{k}$. This situation is standard for quantum mechanics [7] where the reference represents a zero energy and other energy levels are gradually increased by step functions. Such system is represented by following phase structure as:

$$
\begin{aligned}
\tilde{\nu}_{1} & =\nu_{1} \\
\tilde{\nu}_{2} & =\nu_{1}+\nu_{2} \\
\vdots & \\
\tilde{\nu}_{N} & =\nu_{1}+\nu_{2}+\cdots+\nu_{N} .
\end{aligned}
$$

This model corresponds to gradual evolution of complex systems [3, 4]. At the beginning we have only subsystem $S_{1}$. After adding the subsystem $S_{2}$ the correlation yields into encapsulation into the new subsystem $S_{1,2}$. Now we can imagine adding the subsystem $S_{3}$ to subsystem $S_{1,2}$, which plays the role of a reference for subsystem $S_{3}$. We can continue up to subsystem $S_{N}$ that will be dependent on previous encapsulated subsystem $S_{1,2, \ldots, N-1}$. 


\section{Quantum extended models}

The main idea of extended quantum model is to include into quantum superposition not only events itself $A_{i}, i \in\{1,2, \ldots, N\}$ but also $n$ additional events' functions, e.g. $f_{k}\left(A_{1}, \ldots, A_{N}\right), k \in\{i, \ldots, n\}$. Then the modified quantum model can be written:

$$
\begin{aligned}
\psi(A) & =\psi\left(A_{1}\right) \cdot\left|A_{1}\right\rangle+\psi\left(A_{2}\right) \cdot\left|A_{2}\right\rangle+\cdots+ \\
& +\psi\left(A_{N}\right) \cdot\left|A_{N}\right\rangle+\psi\left(f_{1}\left(A_{1}, \ldots, A_{N}\right)\right) \cdot\left|f_{1}\left(A_{1}, \ldots, A_{N}\right)\right\rangle+\cdots+ \\
& +\psi\left(f_{n}\left(A_{1}, \ldots, A_{N}\right)\right) \cdot\left|f_{n}\left(A_{1}, \ldots, A_{N}\right)\right\rangle
\end{aligned}
$$

Such approach brings many possibilities how rapidly extend the dimensionality of complex system.

Example 2 - dimensionality analyze of binary functions In case of $N$ binary events $A \in\{0,1\}, i \in\{1,2, \ldots, N\}$ we have $2^{N}$ different variants of outputs combinations. If we suppose a binary function applied on each events' combination, theoretically we can achieve $2^{2^{N}}$ different variants of functions outputs.

Let us suppose an example with $N=2$ that means 4 combinations of binary events $A_{1}, A_{2} \in\{(0,0),(0,1),(1,0),(1,1)\}$. By application of two dimensional function $f\left(A_{1}, A_{2}\right)$ we can return $4^{2}=16$ variants of different outputs. For $N=3$ we have 8 binary combinations of events $A_{1}, A_{2}, A_{3}$ but 256 possible variants of binary functions. This example demonstrates how fast the number of free parameters increases by adding additional binary functions.

\subsection{Extended intersectional quantum models}

For practical feasibility, we will restrict us only to events' intersections $f_{h}\left(A_{1}, \ldots\right.$, $\left.A_{N}\right)=A_{k} \cap A_{m} \cap \cdots \cap A_{r}, k, m, \ldots, r \in\{1,2, \ldots, N\}$. The extended quantum model can be than rewritten:

$$
\begin{aligned}
\psi(A) & =\psi\left(A_{1}\right) \cdot\left|A_{1}\right\rangle+\psi\left(A_{2}\right) \cdot\left|A_{2}\right\rangle+\cdots+ \\
& +\psi\left(A_{N}\right) \cdot\left|A_{N}\right\rangle+\psi\left(A_{1} \cap A_{2}\right) \cdot\left|A_{1} \cap A_{2}\right\rangle+\cdots+ \\
& +\psi\left(A_{k} \cap A_{m} \cap \cdots \cap A_{r}\right) \cdot\left|A_{k} \cap A_{m} \cap \cdots \cap A_{r}\right\rangle .
\end{aligned}
$$

There are of course other possibilities how to include additional information into quantum model but the intersections seem to be more natural and easily applicable. In this case, it is possible to manipulate with different combinations of events' intersections ( $\otimes$ is Kronecker product [5]):

$$
\begin{aligned}
\left|A_{i}\right\rangle \otimes\left|A_{i} \cap A_{j}\right\rangle & \Rightarrow\left|A_{i} \cap A_{j}\right\rangle \\
\left|A_{i} \cap A_{k} \cap A_{r}\right\rangle \otimes\left|A_{k} \cap A_{r}\right\rangle & \Rightarrow\left|A_{i} \cap A_{k} \cap A_{r}\right\rangle \\
\left|A_{i} \cap A_{k} \cap A_{r}\right\rangle \otimes\left|A_{p} \cap A_{q}\right\rangle & \Rightarrow\left|A_{i} \cap A_{k} \cap A_{r} \cap A_{p} \cap A_{q}\right\rangle .
\end{aligned}
$$

Such logical rules give us mathematical instrument for wave probabilistic interferences that yields to modelling of new multi-dimensional complex systems like quantum entanglement [2]. 


\subsection{Interpretation of extended intersectional quantum models}

A superposition of different events together with some events' intersections yields into the extended intersectional quantum model. We can divide events' intersections into two groups: inner (microscopic) and outer (macroscopic).

Inner (microscopic) intersections represent emergent feature of complex system and are modeled by phase differences of wave probabilistic functions [2]. Due to positive or negative sigs, they could go to either inner attraction or repelling of events. These features yields into well-known quantum modelling [5,9].

Outer (macroscopic) intersections could be seen as the additional observable behavior that could be considered as the new event (quantity) of studied system. Because of macroscopic nature, we need use only classical probability theory that was developed for description of macroscopic phenomena.

For example, the extended quantum model enables modelling links between two different macroscopic intersections through their wave probabilistic phases. Entanglement than can be realized not only among pure events but also among events' intersections.

Example 3 - social model of relation among company employees We can suppose to have $N$ employees $A_{i}, i \in\{1,2, \ldots, N\}$ with the inner links that are defined psychologically, ability to take responsibility, etc. Such characteristic can be scarcely measured. Its observations are limited to inner phase parameters that are extracted only from holistic behavior of the team.

On the other hand, there are outer (macroscopic) links that have strong impacts on holistic system' behavior and are easily identifiable. We can state e.g. family relationships, schoolmates, etc. Such macroscopic links should be taken into our model to catch better details. If I am employee $A_{r} \mathrm{I}$ am influenced by all other employees $A_{k}, k \neq r$ and also by links to additional (macroscopic) employees groups, e.g. $A_{k} \cap A_{c} \cap A_{d}, A_{q} \cap A_{l} \cap A_{t} \cap A_{o}$. Taking into consideration the all relations among studied group of employees the holistic social model can be better specified.

\section{Application of extended intersectional quantum models}

In many practical applications of quantum models, there is a demand for description of complex networks [8-10]. Optimal management of complex systems consists of the best arrangement of all network nodes represented by amplitudes and phases of all components [2]. The applicability of presented approach will be shown on following examples.

Example 4 - incremental/ordering quantum model We can assume events $A_{1}, A_{2}, A_{3}, A_{4}$ represented by probabilities $P\left(A_{1}\right), P\left(A_{2}\right), P\left(A_{3}\right), P\left(A_{4}\right)$ with assigned wave probabilistic functions:

$$
\psi\left(A_{1}\right)=\sqrt{P\left(A_{1}\right)} \cdot e^{j \cdot \varphi_{1}}, \psi\left(A_{2}\right)=\sqrt{P\left(A_{2}\right)} \cdot e^{j \cdot \varphi 2},
$$




$$
\psi\left(A_{3}\right)=\sqrt{P\left(A_{3}\right)} \cdot e^{j \cdot \varphi_{3}}, \psi\left(A_{4}\right)=\sqrt{P\left(A_{4}\right)} \cdot e^{j \cdot \varphi_{4}} .
$$

The union of all events Eq. (6) can be given as follows:

$$
\begin{aligned}
& P\left(A_{1} \cup A_{2} \cup A_{3} \cup A_{4}\right)=\left|\psi\left(A_{1}\right)+\psi\left(A_{2}\right)+\psi\left(A_{3}\right)+\psi\left(A_{4}\right)\right|^{2}= \\
= & P\left(A_{1}\right)+P\left(A_{2}\right)+P\left(A_{3}\right)+P\left(A_{4}\right)+ \\
+ & 2 \cdot \sqrt{P\left(A_{1}\right) \cdot P\left(A_{2}\right)} \cdot \cos \left(\varphi_{2}-\varphi_{1}\right)+2 \cdot \sqrt{P\left(A_{1}\right) \cdot P\left(A_{3}\right)} \cdot \cos \left(\varphi_{3}-\varphi_{1}\right)+ \\
+ & 2 \cdot \sqrt{P\left(A_{1}\right) \cdot P\left(A_{4}\right)} \cdot \cos \left(\varphi_{4}-\varphi_{1}\right)+2 \cdot \sqrt{P\left(A_{2}\right) \cdot P\left(A_{3}\right)} \cdot \cos \left(\varphi_{3}-\varphi_{2}\right)+ \\
+ & 2 \cdot \sqrt{P\left(A_{2}\right) \cdot P\left(A_{4}\right)} \cdot \cos \left(\varphi_{4}-\varphi_{2}\right)+2 \cdot \sqrt{P\left(A_{3}\right) \cdot P\left(A_{4}\right)} \cdot \cos \left(\varphi_{4}-\varphi_{3}\right) .
\end{aligned}
$$

Comparing with classical probabilistic rule Eq. (3), we can extract:

$$
\begin{aligned}
& P\left(A_{1} \cap A_{2}\right)=2 \cdot \sqrt{P\left(A_{1} \cdot A_{2}\right)} \cdot \cos \left(\varphi_{2}-\varphi_{1}\right), \\
& P\left(A_{1} \cap A_{3}\right)=2 \cdot \sqrt{P\left(A_{1} \cdot A_{3}\right)} \cdot \cos \left(\varphi_{3}-\varphi_{1}\right), \\
& P\left(A_{1} \cap A_{4}\right)=2 \cdot \sqrt{P\left(A_{1} \cdot A_{4}\right)} \cdot \cos \left(\varphi_{4}-\varphi_{1}\right), \\
& P\left(A_{2} \cap A_{3}\right)=2 \cdot \sqrt{P\left(A_{2} \cdot A_{3}\right)} \cdot \cos \left(\varphi_{3}-\varphi_{2}\right), \\
& P\left(A_{2} \cap A_{4}\right)=2 \cdot \sqrt{P\left(A_{2} \cdot A_{4}\right)} \cdot \cos \left(\varphi_{4}-\varphi_{2}\right), \\
& P\left(A_{3} \cap A_{4}\right)=2 \cdot \sqrt{P\left(A_{3} \cdot A_{4}\right)} \cdot \cos \left(\varphi_{4}-\varphi_{3}\right) .
\end{aligned}
$$

To obtain the ordering quantum model (Section 3.1 ) we provide transformation and compute following phases:

$$
\begin{aligned}
\tilde{\nu}_{1}=\varphi_{2}-\varphi_{1}=\varphi_{3}-\varphi_{2} & =\varphi_{4}-\varphi_{3}, \\
\tilde{\nu}_{2}=\varphi_{3}-\varphi_{1} & =\varphi_{4}-\varphi_{2}, \\
\tilde{\nu}_{3} & =\varphi_{4}-\varphi_{1} .
\end{aligned}
$$

The phases $\tilde{\nu}_{1}, \tilde{\nu}_{2}, \tilde{\nu}_{3}$ fully describe the quantum ordering model and there is not necessary to provide any approximation.

Example 5 - extended intersectional quantum model Let us use previous example and to add into this model one additional macroscopic intersection $P\left(A_{3} \cap\right.$ $A_{4}$ ) represented by wave probabilistic function:

$$
\psi\left(A_{3} \cap A_{4}\right)=\sqrt{P\left(A_{3} \cap A_{4}\right)} \cdot e^{j \cdot \nu_{3,4}} .
$$

The extended intersectional quantum model can be written in "bra-ket" notation as:

$$
\begin{array}{r}
\psi\left(A_{1}, A_{2}, A_{3}, A_{4}\right)=\psi\left(A_{1}\right) \cdot\left|A_{1}\right\rangle+\psi\left(A_{2}\right) \cdot\left|A_{2}\right\rangle+ \\
+\psi\left(A_{3}\right) \cdot\left|A_{3}\right\rangle+\psi\left(A_{4}\right) \cdot\left|A_{4}\right\rangle+\psi\left(A_{3} \cap A_{4}\right) \cdot\left|A_{3} \cap A_{4}\right\rangle .
\end{array}
$$

The union of all events Eq. (15) can be enlarged:

$$
\begin{aligned}
& P\left(A_{1} \cup A_{2} \cup A_{3} \cup A_{4}\right)=\psi\left(A_{1}, A_{2}, A_{3}, A_{4}\right) \cdot \psi^{*}\left(A_{1}, A_{2}, A_{3}, A_{4}\right)= \\
= & {\left[\psi\left(A_{1}\right) \cdot\left|A_{1}\right\rangle+\psi\left(A_{2}\right) \cdot\left|A_{2}\right\rangle+\psi\left(A_{3}\right) \cdot\left|A_{3}\right\rangle+\psi\left(A_{4}\right) \cdot\left|A_{4}\right\rangle+\right.} \\
& \left.+\psi\left(A_{3} \cap A_{4}\right) \cdot\left|A_{3} \cap A_{4}\right\rangle\right] \cdot\left[\psi^{*}\left(A_{1}\right) \cdot\left|A_{1}\right\rangle^{*}+\psi^{*}\left(A_{2}\right) \cdot\left|A_{2}\right\rangle^{*}+\right. \\
& \left.+\psi^{*}\left(A_{3}\right) \cdot\left|A_{3}\right\rangle^{*}+\psi^{*}\left(A_{4}\right) \cdot\left|A_{4}\right\rangle^{*}+\psi^{*}\left(A_{3} \cap A_{4}\right) \cdot\left|A_{3} \cap A_{4}\right\rangle^{*}\right] .
\end{aligned}
$$




\section{Svítek M.: Quantum multidimensional models of complex systems}

We can use the following "composition" rules:

$$
\begin{gathered}
\quad \psi\left(A_{i}\right) \cdot\left|A_{i}\right\rangle \cdot \psi^{*}\left(A_{j}\right) \cdot\left|A_{j}\right\rangle^{*}+ \\
+\quad \psi\left(A_{j}\right) \cdot\left|A_{j}\right\rangle \cdot \psi^{*}\left(A_{i}\right) \cdot\left|A_{i}\right\rangle^{*}= \\
=\quad \sqrt{P\left(A_{i}\right) \cdot P\left(A_{j}\right)} \cdot \cos \left(\varphi_{i}-\varphi_{j}\right) \cdot\left|A_{i} \cap A_{j}\right\rangle, \\
+\quad \psi\left(A_{i}\right) \cdot\left|A_{i}\right\rangle \cdot \psi^{*}\left(A_{j} \cap A_{k}\right) \cdot\left|A_{j} \cap A_{k}\right\rangle^{*}+ \\
=\quad \sqrt{P\left(A_{j} \cap A_{k}\right) \cdot\left|A_{j} \cap A_{k}\right\rangle \cdot \psi^{*}\left(A_{i}\right) \cdot\left|A_{i}\right\rangle^{*}=} \\
\quad \psi\left(A_{j} \cap A_{k}\right) \cdot \cos \left(\varphi_{i}-\varphi_{j, k}\right) \cdot\left|A_{i} \cap A_{j} \cap A_{k}\right\rangle, \\
+\quad \psi\left(A_{i} \cap A_{k}\right) \cdot\left|A_{i} \cap A_{k}\right\rangle \cdot \psi^{*}\left(A_{i}\right) \cdot\left|A_{i}\right\rangle^{*}= \\
=\quad \sqrt{P\left(A_{i}\right) \cdot P\left(A_{i} \cap A_{k}\right)} \cdot \cos \left(\varphi_{i}-\varphi_{i, k}\right) \cdot\left|A_{i} \cap A_{k}\right\rangle .
\end{gathered}
$$

The probabilistic union of enlarged intersectional model can be computed:

$$
\begin{aligned}
P\left(A_{1} \cup A_{2} \cup A_{3} \cup A_{4}\right) & =\left|\psi\left(A_{1}\right)+\psi\left(A_{2}\right)+\psi\left(A_{3}\right)+\psi\left(A_{4}\right)+\psi\left(A_{3} \cap A_{4}\right)\right|^{2}= \\
& =P\left(A_{1}\right)+P\left(A_{2}\right)+P\left(A_{3}\right)+P\left(A_{4}\right)+P\left(A_{3} \cap A_{4}\right)+ \\
& +2 \cdot \sqrt{P\left(A_{1}\right) \cdot P\left(A_{2}\right)} \cdot \cos \left(\varphi_{2}-\varphi_{1}\right)+ \\
& +2 \cdot \sqrt{P\left(A_{1}\right) \cdot P\left(A_{3}\right)} \cdot \cos \left(\varphi_{3}-\varphi_{1}\right)+ \\
& +2 \cdot \sqrt{P\left(A_{1}\right) \cdot P\left(A_{4}\right)} \cdot \cos \left(\varphi_{4}-\varphi_{1}\right)+ \\
& +2 \cdot \sqrt{P\left(A_{2}\right) \cdot P\left(A_{3}\right)} \cdot \cos \left(\varphi_{3}-\varphi_{2}\right)+ \\
& +2 \cdot \sqrt{P\left(A_{2}\right) \cdot P\left(A_{4}\right)} \cdot \cos \left(\varphi_{4}-\varphi_{2}\right)+ \\
& +2 \cdot \sqrt{P\left(A_{3}\right) \cdot P\left(A_{4}\right)} \cdot \cos \left(\varphi_{4}-\varphi_{3}\right)+ \\
& +2 \cdot \sqrt{P\left(A_{1}\right) \cdot P\left(A_{3} \cap A_{4}\right)} \cdot \cos \left(\varphi_{3,4}-\varphi_{1}\right)+ \\
& +2 \cdot \sqrt{P\left(A_{2}\right) \cdot P\left(A_{3} \cap A_{4}\right)} \cdot \cos \left(\varphi_{3,4}-\varphi_{2}\right)+ \\
& +2 \cdot \sqrt{P\left(A_{3}\right) \cdot P\left(A_{3} \cap A_{4}\right)} \cdot \cos \left(\varphi_{3,4}-\varphi_{3}\right)+ \\
& +2 \cdot \sqrt{P\left(A_{4}\right) \cdot P\left(A_{3} \cap A_{4}\right)} \cdot \cos \left(\varphi_{3,4}-\varphi_{4}\right) .
\end{aligned}
$$

In addition of intersections Eq. (16), $P\left(A_{3} \cap A_{4}\right)$ was extended to:

$$
\begin{aligned}
\tilde{P}\left(A_{3} \cap A_{4}\right)=P\left(A_{3} \cap A_{4}\right)+ & 2 \cdot \sqrt{P\left(A_{3}\right) \cdot P\left(A_{3} \cap A_{4}\right)} \cdot \cos \left(\varphi_{3,4}-\varphi_{3}\right)+ \\
& +2 \cdot \sqrt{P\left(A_{4}\right) \cdot P\left(A_{3} \cap A_{4}\right)} \cdot \cos \left(\varphi_{3,4}-\varphi_{4}\right),
\end{aligned}
$$

where $\tilde{P}\left(A_{3} \cap A_{4}\right)$ is modified extended intersection probability $P\left(A_{3} \cap A_{4}\right)$ enriched with inner links to events $A_{3}, A_{4}$.

The third order intersections appeared due to added wave function Eq. (20):

$$
\begin{aligned}
& P\left(A_{1} \cap A_{3} \cap A_{4}\right)=2 \cdot \sqrt{P\left(A_{1}\right) \cdot P\left(A_{3} \cap A_{4}\right)} \cdot \cos \left(\varphi_{3,4}-\varphi_{1}\right), \\
& P\left(A_{2} \cap A_{3} \cap A_{4}\right)=2 \cdot \sqrt{P\left(A_{2}\right) \cdot P\left(A_{3} \cap A_{4}\right)} \cdot \cos \left(\varphi_{3,4}-\varphi_{2}\right) .
\end{aligned}
$$


In a similar way, more variants of macroscopic intersections can be included into extended intersectional quantum model. The more intersections (pieces of information), the more available additional parameters and the more possibility to model multi-dimensional complex system.

\section{Conclusion}

The main goal of the paper was to find the way to overcome the dimensionality problem of quantum models. The proposed solution supposes including events' combinations (function of events) into mass parallel quantum model as the additional macroscopic state. For better feasibility, we concentrated only on the intersectional macroscopic models (intersections of selected events). The events' intersections could be easily interpreted and logical rules, how to work with them, were developed.

For complete description of all correlations (links) among events $A_{i}, i \in\{1,2, \ldots$, $N\}$ we need to specify at least $\frac{N \cdot(N+1)}{2}$ free parameters. This corresponds to requirements how many different intersections we need to include into the extended intersectional quantum model. We revealed on illustrative example how to incorporate new information into the extended model. The more intersections the better model we can build.

The benefit of the extended model is that a well-known entanglement cannot be only among the pure events but also among the different intersections or among the combinations of intersections and pure events. These features bring new possibilities for modelling especially soft systems with enormous links and interconnections.

In quantum physic, moduli represent typically energy [7]. In our approach, the mathematical instrument of wave probabilities has, therefore, much broader applications than in physics $[12,13]$. In system sciences, for example, we can evaluate by quantum models other system features like the ability to create alliances [8], the ability for adaptation (the quickest response to changes) [6], etc. The presented methodology can be further enlarged not only to entanglement among pure events and events' functions, but also it can add an enlargement of different system processes and their functions. If we imagine the complexity of such a model [11], we are coming closer to Kaufmann tissue [6].

\section{Acknowledgement}

This work was supported by the Project AI \& Reasoning CZ.02.1.01/0.0/0.0/15_003/ 0000466 and the European Regional Development Fund.

\section{References}

[1] SVÍTEK M., KOSHELEVA O., KREINOVICH V., NGUYEN T. N. Why Quantum (Wave Probability) Models Are a Good Description of Many Non-quantum Complex Systems, and How to Go Beyond Quantum Models), pp. 168-175, In: Kreinovich et al. (Eds.): Beyond Traditional Probabilistic Methods in Economics, Springer Nature Switzerland AG 2019, SCI 809, 2019, doi: 10.1007/978-3-030-04200-4_13. 


\section{Svítek M.: Quantum multidimensional models of complex systems}

[2] SVÍTEK M. Towards complex system theory, Tutorial, Neural Network World, 2015, 25(1), pp. 5-33, ISSN 1210-0552.

[3] SVÍTEK M.: Applying Wave Probabilistic Functions for Dynamic System Modeling, IEEE transactions on systems man and cybernetics, part c-applications and reviews, 41(5), pp. 674-681, 2011, doi: 10.1109/TSMCC. 2010.2093127.

[4] SVÍTEK M. Wave probabilistic functions for quantum cybernetics, 2012, IEEE transactions on systems man and cybernetics, part c-applications and reviews. 42(2), pp. 233-240, 2012, doi: 10.1109/TSMCC.2011.2115237.

[5] VEDRAL V. Introduction to Quantum Information Science, Oxford University Press, 2006.

[6] KAUfFMAn S. Autonomous Agents, in John D. Barrow, P.C.W. Davies, and C.L. Harper Jr., eds., Science and Ultimate Reality: Quantum Theory, Cosmology, and Complexity, Cambridge University Press. 2004. ISBN 9780521831130.

[7] FEYNMAN R., LEIGHTON R., SANDS M. Feynman lectures of physics, Addison Wesley Longman, Inc.. USA, 1966.

[8] VOTRUBA Z., NOVÁK M. Alliance Approach to the Modelling of Interfaces in Complex Heterogenous Objects. Neural Network World, 2010, 20(5), pp 609-619.

[9] SVÍTEK M. Physics-Information Analogies, Neural Network World, 2018, 28(6), pp. 535550, doi: $10.14311 / \mathrm{NNW} .2018 .28 .030$

[10] MOOS P., SVÍTEK M., NOVÁK M., VOTRUBA Z. Information Model of Resonance Phenomena in Brain Neural Networks. Neural Network World, 2018, 28(3), pp. 225-239, ISSN 1210-0552.

[11] SVÍTEK M.: Wave probabilistic information power, In: Neural Network World 2011, 21(3), pp. 269-276, ISSN 1210-0552.

[12] MALINOVSKÝ, V.: System Macromodel of Agricultural Building with Aim to Energy Consumption Minimization, AGRIS on-line Papers in Economics and Informatics, 10(1), 2018, pp. 25-35. ISSN 1804-1930. doi: 10.7160/aol.2018.100103.

[13] BOUCHNER P., HAJNY M., NOVOTNY S., et al. Car simulation and virtual environments for investigation of driver behavior, Neural Netw. World, 15(2), pp. 149-163, 2005. 